\title{
Rheumatoid Arthritis - Prevention and Treatment with a Plant-Based Diet
}

\author{
Stewart Rose* and Amanda Strombom \\ Plant-Based Diets in Medicine, USA
}

Submission: September 26, 2018; Published: October 05, 2018

*Corresponding author: Stewart Rose, Vice President, Plant based Diets in Medicine, 12819 SE 38 ${ }^{\text {th }}$ St, \#427, Bellevue, WA 98006, USA, Tel: 4255312829; Email: Contact@pbdmedicine.org

\begin{abstract}
Rheumatoid arthritis has no cure, so long term treatment is indicated. An individual's dietary choices greatly influence the progression of chronic autoimmune rheumatic diseases. This review shows that the plant-based diet has good scientific evidence of safety and efficacy for both prevention and treatment of rheumatoid arthritis. Studies have shown significant improvements in specific symptoms, such as number of tender joints, Ritchie's articular index, number of swollen joints, pain score, duration of morning stiffness, grip strength, and improved laboratory values such as sed rate (ESR), C-reactive protein, and rheumatic factor. Patients placed on a plant-based diet also have a beneficial shift in intestinal microbiota, which correlates with clinical improvement. With respect to prevention, those following a plant-based diet experience a reduction in risk of rheumatoid arthritis by about $50 \%$.

RA patients should be advised that a plant-based diet that includes appropriate amounts of carbohydrate, especially dietary fiber, is important for maintaining the symbiosis of intestinal flora, which could be beneficial for preventing autoimmunity. As disease severity worsens, individuals with RA may experience functional decline that can impact dietary intake. New healthy plant-based convenience foods are a good choice for such patients. Treatment with a plant-based diet is affordable for the patient, has no adverse reactions and no contraindications, and it can be combined with any of the standard treatments. For mild cases it may suffice as a monotherapy. For moderate and severe cases, it may serve as an adjunct, allowing dosage reductions thus lessening the costs and side effects.
\end{abstract}

Keywords: Rheumatoid Arthritis; Autoimmunity; Rheumatic; Plant-based diet; Vegan; Vegetarian; Intestinal flora; Microbiota; Dietary fiber; Dietary modification

\section{Introduction}

Rheumatoid arthritis (RA) is a chronic systemic inflammatory disease of unknown etiology. There is no cure, so long term treatment is indicated. Medication-based therapies comprise several classes of agents, including nonsteroidal anti-inflammatory drugs (NSAIDs), non-biologic and biologic disease modifying anti-rheumatic drugs (DMARDs), immunosuppressants, and corticosteroids. Other standard treatments include physical therapy and surgery.Surveys have shown that a substantial proportion of people with RA will try complementary and alternative interventions, perhaps reflecting the lack of complete satisfaction with conventional approaches, and a desire to help themselves [1]. In 1989, Arthritis Care noted that more than 50\% of the Arthritis Care members who were surveyed, had invested in "unorthodox medicines, substances, or treatments (including diets), during the prior six months".

Today, with increased access to health care information has come a growing demand for safe, cost-effective and easy to administer therapies. While a number of purported treatments have questionable or no research behind them, one of these socalled "unorthodox treatments" does [2]. An individual's dietary choices can greatly influence the progression and manifestation of chronic autoimmune rheumatic diseases. In light of these effects, it makes sense that the search for additional therapies to attenuate such diseases would include investigations into dietary modifications [3].

Dietary interventions have a widespread appeal for both patients as well as clinicians due to factors including affordability, accessibility, and presence of scientific evidences that demonstrate substantial benefits in reducing disease symptoms such as pain, joint stiffness, swelling, tenderness and associated disability with disease progression [4].

\section{Epidemiology}

There have been few studies on the risk of RA in relation to vegetarian status.However, one good-sized study showed that non-vegetarian women had a $57 \%$ increased risk of RA, and semi-vegetarians an increased risk of $16 \%$, when compared with vegetarian women. Non-vegetarian men showed an increased risk of $50 \%$ and semi-vegetarian men an increased risk of $14 \%$ [5]. These results are especially notable considering that the non-vegetarians in this study already had a relatively low consumption of meat. 


\section{Orthopedics and Rheumatology Open Access Journal (OROAJ)}

\section{Interventional Studies}

Many doctors have anecdotally noted an association between the consumption of animal-derived foods, especially meat, and Rheumatoid Arthritis [6]. A meta-study on RA looked at studies on the effects of short-term modified fasting followed subsequently with plant-based diets lasting at least three months. The pooling of these studies showed a statistically and clinically significant beneficial long-term effect. Thus, available evidence suggests that fasting followed by vegetarian diets might be useful in the treatment of RA [7].

The effect of a one-year study on RA patients of brief (710 day) modified fasting, followed first by a vegan diet and then by a vegetarian diet was assessed in a randomized, single blind controlled trial.After four weeks, the diet group showed a significant improvement in the number of tender joints, Ritchie's articular index, number of swollen joints, pain score, duration of morning stiffness, grip strength, erythrocyte sedimentation rate, $\mathrm{C}$ reactive protein, white blood cell count, and a health assessment questionnaire score. The improvements were still present at the end of one year. A significant drop in the levels of intestinal Proteus Mirabilis was observed [8].

In a follow-up study of the same patients two years later, pain score, duration of morning stiffness, Stanford Health Assessment Questionnaire index, number of tender joints, Ritchie's articular index, number of swollen joints, ESR and platelet count all maintained their improvement in patients who were responders.Interestingly, most patients who were originally in the vegetarian group, but switched back to their usual diet, reported an increase in disease symptoms after intake of meat [9]. A separate one-year study of RA patients placed on a vegetarian diet, and focusing on clinical laboratory values, showed significant improvements in leukocyte count, IgM, RF (Rheumatic Factor), and the complement components C3 and C4, along with patient symptoms [10].

It usually takes several months for a plant-based diet to reach full effect in RA. However, one study showed improvements in pain, joint swelling, severity in morning stiffness and limitation in function in only 4 weeks. There were also non-significant trends in the reduction of C-reactive protein and Rheumatic factor. Based on the results of other studies, these might have become significant with further time [11]. Studies have noted a link between vegan diets and protection against other autoimmune diseases. For example, an analysis of an Adventist cohort found that a vegan diet, but not a vegetarian diet, was associated with a lower risk of hypothyroid disease [12].

Several researchers have examined the role of gut bacteria in RA disease activity [13-17]. Researchers Ling and Hänninen tested subjects on both a conventional Western diet and a vegan diet for one month, in order to determine the shift in intestinal flora. They found that four fecal hydrolytic enzymes, associated with toxic and inflammatory products, diminished during consumption of the vegan diet. However, these changes in fecal urease, choloylglycine hydrolase, $\beta$-glucuronidase and $\beta$-glucosidase, disappeared within two weeks of resuming a conventional diet. The authors attribute these reductions in fecal enzymes not only to the activity of bacteria during the dietary shift, but also to the high fiber content of the vegan diet which can affect fecal weight, transit time and bacterial metabolism [18].

RA patients have been found to have higher levels of Proteus mirabilis antibodies, when compared with healthy controls or subjects with other diseases. The subjects from the vegetarian diet study had a significantly lower mean antibody level against Proteus mirabilis, which was correlated significantly with the measured decrease in disease activity [19]. This suggests that the improvement in RA disease activity may be related to the effects of the vegan diet on the presence of gut bacteria, such as Proteus mirabilis, and the body's response to such bacteria.

The possibility that a vegan diet can induce a rapid change in gut profile was supported by studies of rheumatoid arthritis patients, in which a one-month switch to a vegan diet was sufficient to significantly alter the fecal microflora, as determined by stool sample gas-liquid chromatography profiles of bacterial cellular fatty acids [20,21]. Peltonen et al. [20] conducted a study of RA patients and found a significant change in intestinal flora after a one-year shift from a conventional diet to a vegan and then a lactovegetarian diet. They also noted a significant difference between the fecal flora of test subjects in the high improvement group and the low improvement group, suggesting a direct connection between gut profiles and levels of disease activity [22].

To further test the role of diet-induced changes in levels of various intestinal flora on rheumatoid arthritis activity, 43 RA patients were randomly assigned to either a raw vegan diet rich in lactobacilli, or an omnivorous diet. After one month, there was a significant change in the fecal flora of the 18 subjects in the vegan diet group who completed the study; no such change was found in the omnivore control group. Importantly, the vegan diet also induced a decrease in disease activity in some of the RA patients, leading the authors to conclude that changes in the intestinal flora are associated with diet-induced changes in disease activity [20].

Kjeldsen-Kragh et al. [19] followed upon their work by putting rheumatoid arthritis patients on a fast followed by 3.5 months of a vegan diet, followed by a 9-month lactovegetarian diet [23]. Subjects in the vegan then vegetarian diet group improved significantly over those maintained on an omnivorous diet. Similar to other studies, the authors found that subjects' fecal flora during times of clinical improvement differed significantly from times of no or minor improvements. Others have found that a raw vegan diet rich in lactobacilli and fiber decreased symptoms of rheumatoid arthritis, suggesting that the probiotic lactobacilli, among other components of a raw vegan 
diet, may be helpful to RA patients [24-26]. One way that these bacteria are helpful is that they regulate the $\mathrm{T}$ cell phenotype and $T$ cell mediated immunity [27].

Caution is warranted in interpreting bacteriologic studies on vegan diets and RA. Although diet-induced modification in intestinal flora, and an associated reduction in inflammation severity, may be a contributing factor to the improvements seen in RA patients, it is important to note that other features of a vegan diet have been credited with alleviating RA symptoms among vegan diet adherents. These include an increase in fruit, vegetable and fiber intake, a reduction in saturated fat and caloric intake, improved antioxidant levels, weight loss, and a reduction in food allergies and intolerances $[17,28,29]$.

It has been observed that vegetarians consume enough foods naturally containing salicylates to have an anti-inflammatory effect. The presence of salicylate in the blood of patients placed on a vegetarian diet was found at concentrations that are known to inhibit the transcription of COX 2, a key inflammatory enzyme in various pathologies. An emphasis on those foods highest in salicylate might enhance the therapeutic effect of a plant-based diet and warrants further investigation [30]. The low-fat vegan diet and diets rich in unsaturated fat (such as plant-based oils) or probiotics have positive effects at alleviating pain and on inflammation markers [31]. There was much hope for the role for Omega 3 fatty acids from fish oil. However, clinical studies on supplementation of $\omega-3$ fatty acids have not supported the expectations [32-37].

\section{Reduction of Risk of Coronary Artery Disease (CAD)}

Patients with rheumatoid arthritis (RA) have increased cardiovascular disease and mortality [38-40]. Several recent studies indicate an increased prevalence not only of cardiovascular disease (CVD) but also of atherosclerosis as determined by ultrasound tomography of carotid arteries $[38,41,42]$. The underlying mechanisms causing this increased risk are not wholly clarified but inflammation and disease duration are suggested to be of importance [43-46].

One study investigated the effects of a vegan diet, in patients with rheumatoid arthritis (RA), on the blood lipids: oxidized low-density lipoprotein (oxLDL) and on natural atheroprotective antibodies against phosphorylcholine (anti-PCs). The study examined the effects of intervention using a gluten-free vegan diet on patients with active RA. They were randomly assigned to either a vegan diet or a well-balanced non-vegan diet for one year. The gluten-free vegan diet induced significantly lower body mass index (BMI), low density lipoprotein (LDL), ox LDL, total cholesterol, and higher anti-PC IgM than control diet. Triglycerides and high-density lipoprotein did not change, since this was not a low-fat vegan diet. Therefore, a vegan diet in patients with RA induces changes that are potentially atheroprotective and anti-inflammatory, including decreased LDL and oxLDL levels, and raised anti-PC IgM and IgA levels [47].

\section{Clinical Considerations}

Rheumatoid arthritis (RA) afflicts approximately 1.5 million American adults and is a major cause of disability. As disease severity worsens, individuals with RA may experience functional decline that can impact dietary intake. The diet quality of many individuals with RA needs improvement and may be related to functional disability associated with RA. Healthcare providers should encourage individuals with RA to meet dietary guidelines and maintain a healthy diet. Moreover, healthcare providers should be aware of the potential impacts of functional disability on diet quality in individuals with RA [48].

Patients with rheumatoid arthritis often have trouble preparing foods that require manual dexterity and strength. These patients should be counseled to purchase the new healthy plant-based convenience foods that are now widely available. These foods are usually higher in fiber and lower in sugar, fat, sodium and calories than ordinary convenience foods [49]. RA patients should be advised that a plant-based diet that includes appropriate amounts of carbohydrate, especially dietary fiber, is important for maintaining the symbiosis of intestinal flora, which could be beneficial for preventing autoimmunity [27]. Active participation of the patient and family in the design and implementation of the therapeutic program helps ensure compliance, as does explaining the rationale for dietary treatment.

This treatment may be sufficient as a monotherapy in mild cases or can be used as an adjunct to standard treatments in moderate and severe cases. Dosages may be able to be titrated down as the clinical effects of the diet manifest themselves.

\section{Discussion}

Optimal care of patients with rheumatoid arthritis consists of an integrated approach that includes both pharmacologic and non-pharmacologic therapies. Medications have side effects which must be managed and are costly to the patient. Some of the non-pharmacologic treatments are available for this disease include physical therapy and surgery [50]. A plant-based diet should be added to this list.

Studies show that not only is a plant-based diet safe and efficacious for the prevention and treatment of Rheumatoid Arthritis, it has several advantages in its favor. It has no adverse effects, no contraindications, and it's very affordable for the patient.It can be combined with any standard treatment and is likely to synergize treatments effects with them. It is safe in the long term and has been shown to reduce the risk of comorbidities, such as coronary artery disease, in rheumatoid arthritis patients. Further research should focus on the most effective dietary elements within plant-based diets.

\section{Conflict of Interest}

The authors state no conflicts of interest or funding sources. 


\section{References}

1. Ernst E (2004) Musculoskeletal conditions and complementary/ alternative medicine. Best Pract Res Clin Rheumatol 18(4): 539-556.

2. Darlington L (1991) Dietary therapy for arthritis. Rheumatic Disease Clinics of North America 17(2): 273-286.

3. Dahan S, Segal Y, Shoenfeld Y (2017) Dietary factors in rheumatic autoimmune diseases: a recipe for therapy? Nat Rev Rheumatol J 13(6): 348-358

4. Khanna S, Jaiswal K, Gupta B (2017) Managing Rheumatoid Arthritis with Dietary Interventions. Front Nutr 4: 52.

5. Fraser G (1999) Associations between diet and cancer, ischemic heart disease, and all-cause mortality in non-Hispanic white California Seventh-day Adventists. American Journal of Clinical Nutrition 70(3): 532s-538s.

6. Kutlu A, Oztürk S, Taşkapan O, Onem Y, Kiralp MZ, et.al. (2010) Meatinduced joint attacks, or meat attacks the joint: rheumatism versus allergy. Nutrition in Clinical Practice 25(1): 90-91.

7. Müller H, de Toledo F, Resch K (2001) Fasting followed by vegetarian diet in patients with rheumatoid arthritis: a systematic review. Scand J Rheumatol 30(1): 1-10.

8. Kjeldsen-Kragh J, Haugen M, Borchgrevink C, Laerum E, Eek M, et.al. (1991) Controlled trial of fasting and one-year vegetarian diet in rheumatoid arthritis. Lancet 338(8772): 899-902.

9. Kjeldsen-Kragh J, Haugen M, Borchgrevink C, Forre O (1994) Vegetarian diet for patients with rheumatoid arthritis--status: two years after introduction of the diet. Clin Rheumatol 13(3): 475-482.

10. Kjeldsen-Kragh J, Mellbye O, Haugen M, Mollnes TE, Hammer HB, et al. (1995) Changes in laboratory variables in rheumatoid arthritis patients during a trial of fasting and one-year vegetarian diet. Scand J Rheumatol 24(2): 85-93.

11. McDougall J, Bruce B, Spiller G, Westerdahl J, McDougall M (2002) Effects of a very low-fat, vegan diet in subjects with rheumatoid arthritis. J Altern Complement Med 8(1): 71-75.

12. Tonstad S, Nathan E, Oda K, Fraser G (2013) Vegan Diets and Hypothyroidism. Nutrients 5(11): 4642-4652.

13. Hazenberg M, Klasen I, Kool J, Ruseler-van Embden JG, Severijnen AJ (1992) Are intestinal bacteria involved in the etiology of rheumatoid arthritis? APMIS 100(1): 1-9.

14. Deighton C, Gray J, Bint A, Walker DJ (1992) Anti-Proteus antibodies in rheumatoid arthritis same-sexed sibships. Br J Rheumatol 31(4): 241-245.

15. Ebringer A, Ptaszynska T, Corbett M, Wilson C, Macafee Y, et al. (1985) Antibodies to Proteus in Rheumatoid Arthritis. Lancet 326(8450): 305-307.

16. Rogers P, Hassan J, Bresnihan B, Feighery C, Whelan A (1988) Antibodies to Proteus in rheumatoid arthritis. Br J Rheumatol 27 Suppl 2: $90-94$.

17. Glick-Bauer M, Yeh MC (2014) The Health Advantage of a Vegan Diet: Exploring the Gut Microbiota Connection. Nutrients 6(11): 4822-4838.

18. Ling W, Hänninen $O$ (1992) Shifting from a conventional diet to an uncooked vegan diet reversibly alters fecal hydrolytic activities in humans. J Nutr 122(4): 924-930.

19. Kjeldsen-Kragh J, Rashid T, Dybwad A, Ebringer A (1995) Decrease in anti-Proteus mirabilis but not anti-Escherichia coli antibody levels in rheumatoid arthritis patients treated with fasting and a one year vegetarian diet. Ann Rheum Dis 54(3): 221-224.
20. Peltonen R, Nenonen M, Helve T, Hänninen O, Toivanen P, et al. (1997) Faecal microbial flora and disease activity in rheumatoid arthritis during a vegan diet. Br J Rheumatol 36(1): 64-68.

21. Peltonen R, Ling W, Hänninen O, Eerola E (1992) An uncooked vegan diet shifts the profile of human fecal microflora: Computerized analysis of direct stool sample gas-liquid chromatography profiles of bacterial cellular fatty acids. Appl Environ Microbiol 58(11): 3660-3666.

22. Peltonen R, Kjeldsen-Kragh J, Haugen M, Eerola E (1994) Changes of faecal flora in rheumatoid arthritis during fasting and one-year vegetarian diet. Br J Rheumatol 33(7): 638-643.

23. Kjeldsen-Kragh J (1999) Rheumatoid arthritis treated with vegetarian diets. American Journal of Clinical Nutrition. 70(3 Suppl): 594s-600s.

24. Nenonen M, Helve T, Rauma A, Hänninen 00 (1998) Uncooked, lactobacilli-rich, vegan food and rheumatoid arthritis. Br J Rheumatol 37(3): 274-281.

25. Hänninen O, Rauma A, Kaartinen K, Nenonen M (1999) Vegan diet in physiological health promotion.. Acta Physiol Hung 86(3-4): 171-180.

26. Hänninen 0 , Kaartinen $K$, Rauma A, Nenonen M, Törrönen R, et al. (2000) Antioxidants in vegan diet and rheumatic disorders. Toxicology 155(1-3): 45-53.

27. Masuko K (2018) A Potential Benefit of "Balanced Diet" for Rheumatoid Arthritis. Front Med (Lausanne) 5: 141.

28. Smedslund G, Byfuglien M, Olsen S, Hagen K (2010) Effectiveness and safety of dietary interventions for rheumatoid arthritis: A systematic review of randomized controlled trials. J Am Diet Assoc 110(5): 727735.

29. Hafström I, Ringertz B, Spångberg A, von Zweigbergk L, Brannemark S, et.al. (2001) A vegan diet free of gluten improves the signs and symptoms of rheumatoid arthritis: The effects on arthritis correlate with a reduction in antibodies to food antigens. Rheumatology (Oxford) 40(10): 1175-1179.

30. Blacklock C, Lawrence J, Wiles D, Malcolm EA, Gibson IH, et.al. (2001) Salicylic acid in the serum of subjects not taking aspirin. Comparison of salicylic acid concentrations in the serum of vegetarians, nonvegetarians, and patients taking low dose aspirin. J Clin Pathol 54(7): 553-555.

31. Badsha H (2018) Role of Diet in Influencing Rheumatoid Arthritis Disease Activity. Open Rheumatol J 12: 19-28.

32. Haugen M, Fraser D, Forre $O$ (1999) Diet therapy for the patient with rheumatoid arthritis? Rheumatology (Oxford) 38(11): 1039-1044.

33. Pullman-Mooar S, Laposata M, Lem D, Holman RT, Leventhal LJ, et.al. (1990) Alteration of cellular fatty acid profile and the production of eicosanoids in human monocytes by gamma-linolenic acid. Arthritis and Rheumatism 33(10): 1526-1533.

34. Kremer J, Jubiz W, Michalek A, Rynes RI, Bartholomew LE, et al. (1987) Fish-Oil Fatty Acid Supplementation in Active Rheumatoid Arthritis: A Double-Blinded, Controlled, Crossover Study. Ann Intern Med 106(4): 497-503.

35. Tate G, Mandell B, Laposata M, Ohliger D, Baker DG, et al. (1989) Suppression of acute and chronic inflammation by dietary gamma linolenic acid. J Rheumatol 16(6): 729-734.

36. Endres S, Ghorbani R, Kelley V, Georgilis K, Lonnemann G, et al. (1989) The effect of dietary supplementation with n-3 polyunsaturated fatty acids on the synthesis of interleukin- 1 and tumor necrosis factor by mononuclear cells. N Engl J Med 320(5): 265-271.

37. Kjeldsen-Kragh J, Lund J, Riise T, Finnanger B, Haaland K, et al. (1992) Dietary omega-3 fatty acid supplementation and Naproxen treatment in patients with rheumatoid arthritis. J Rheumatol 19(10): 1531-1536. 
38. Frostegård J (2005) Atherosclerosis in patients with autoimmune disorders. Arteriosclerosis, Thrombosis and Vascular Biology 25(9): 1776-1785.

39. Solomon D, Karlson E, Rimm E, Cannuscio CC, Mandi LA, et al. (2003) Cardiovascular morbidity and mortality in women diagnosed with rheumatoid arthritis. Circulation 107(9): 1303-1307.

40. Wolfe F, Freundlich B, Straus W (2003) Increase in cardiovascular and cerebrovascular disease prevalence in rheumatoid arthritis.. Rheumatol 30(1): 36-40.

41. Park Y, Ahn C, Choi H, Lee SH, In BH, et al. (2002) Atherosclerosis in rheumatoid arthritis: morphologic evidence obtained by carotid ultrasound. Arthritis Rheum 46(7): 1714-1719.

42. Jonsson S, Backman C, Johnson O, Karp K, Lundström E, et al. (2001) Increased prevalence of atherosclerosis in patients with medium term rheumatoid arthritis. J Rheumatol 28(12): 2597-2602.

43. Del Rincón I, Williams K, Stern M, Freeman GL, O’Leary DH, et al. (2003) Association between carotid atherosclerosis and markers of inflammation in rheumatoid arthritis patients and healthy subjects Arthritis Rheum 48(7): 1833-1840.

44. Nagata-Sakurai M, Inaba M, Goto H, Kumeda Y, Furumitsu Y, et al (2003) Inflammation and bone resorption as independent factors of accelerated arterial wall thickening in patients with rheumatoid arthritis. Arthritis Rheum 48(11): 3061-3067.
45. Wållberg-Jonsson S, Cvetkovic J, Sundqvist K, Lefvert AK, RantapääDahlqvist S (2002) Activation of the immune system and inflammatory activity in relation to markers of atherothrombotic disease and atherosclerosis in rheumatoid arthritis. J Rheumatol 29(5): 875-882.

46. Del Rincón I, O’Leary D, Freeman G, Escalante A (2007) Acceleration of atherosclerosis during the course of rheumatoid arthritis. Atherosclerosis 195(2): 354-360.

47. Elkan A, Sjöberg B, Kolsrud B, Ringertz B, Hafström I, etal. (2008) Glutenfree vegan diet induces decreased LDL and oxidized LDL levels and raised atheroprotective natural antibodies against phosphorylcholine in patients with rheumatoid arthritis: a randomized study. Arthritis Res Ther 10(2): R34.

48. Berube L, Kiely M, Yazici Y, Woolf K (2017) Diet quality of individuals with rheumatoid arthritis using the Healthy Eating Index (HEI)-2010. Nutr Health 23(1): 17-24

49. Mizukami Y, Matsui T, Tohma S, Masuko K (2017) Distinct patterns of dietary intake in different functional classes of patients with rheumatoid arthritis. Top Clin Nutr 32(2): 141-151.

50. National Collaborating Centre for Chronic Conditions (UK) (2009) Rheumatoid Arthritis: National Clinical Guideline for Management and Treatment in Adults. Royal College of Physicians, London, UK.

\section{Your next submission with Juniper Publishers} will reach you the below assets

- Quality Editorial service

- Swift Peer Review

- Reprints availability

- E-prints Service

- Manuscript Podcast for convenient understanding

- Global attainment for your research

- Manuscript accessibility in different formats ( Pdf, E-pub, Full Text, Audio)

- Unceasing customer service

Track the below URL for one-step submission https://juniperpublishers.com/online-submission.php 